\title{
SURVEY OF DEVICE CALIBRATION TECHNIQUES FOR FINGERPRINTING LOCALIZATION ALGORITHMS
}

\begin{abstract}
In the paper device calibration algorithms for indoor positioning systems based on fingerprinting localization framework are discussed and compared. Device calibration should play important role in development of localization systems based on Wi-Fi since heterogeneous devices have different receiver parameters. This fact causes that the positioning system cannot provide position estimates with sufficient accuracy. In this paper different calibration approaches will be described and two state of art automatic calibration techniques will be compared in the simulations.
\end{abstract}

Keywords: Indoor positioning, localization, fingerprinting, device calibration.

\section{Introduction}

In the recent years many localization systems for indoor positioning were proposed and developed [1]. The main reason is that satellite navigation systems, which are widely used, are not feasible for use in the indoor environment [2]. This is caused by the fact that signals from the satellites are highly attenuated by the building walls and ceilings, so the receiver is not able to accurately estimate its position [3]. This leads many research teams to work on localization systems based on wireless technologies [4 and 5] e.g. GSM, UMTS, ZigBee, UWB and Wi-Fi.

The most popular indoor localization systems are based on Wi-Fi (IEEE $802.11 \mathrm{~b} / \mathrm{g}$ ) technology in combination with fingerprinting approach. The main advantages are that there is no need to build new infrastructure in order to deploy the positioning system and that fingerprinting approach seems to be immune to the multipath phenomenon. Most of fingerprinting based localization systems uses RSS measurements to create a radio map and estimate position. Measurements of RSS can be performed on all devices without any additional modification [6].

However, the use of RSS measurements has one huge drawback. Due to lack of standardization of receivers each device has different parameters and antenna gain. These differences are not only caused by different hardware implemented in devices, but can be also caused by the use of different software. For the successful deployment of the localization system based on Wi-Fi and fingerprinting framework, it is important to use device calibration. Calibration should decrease negative impact of receiver differences on the functionality and accuracy of the localization system.

In this paper we will describe the state of art methods for device calibration in indoor positioning systems based on fingerprinting framework and test proposed methods in order to find optimal solution for device calibration. We assume that optimal calibration technique should provide the same positioning accuracy for different devices without interaction with the user.

The rest of the paper is organized as follows; in the next section the state-of-art fingerprinting localization algorithms will be described. In section 3 device calibration methods will be described and compared. Simulation model used for comparison of chosen calibration methods and simulation scenarios will be introduced in section 4. Results of simulations will be shown in section 5 and section 6 will conclude the paper.

\section{Fingerprinting localization}

The fingerprinting localization technique consists of two phases, the first phase is called offline or training phase and the second is called online phase. During the offline phase a radio map is created and stored in the database. During the online phase the position of mobile device is estimated using localization algorithm and radio map database.

\section{A Offline phase}

The radio map construction process (Fig. 1) can be divided into three main steps. The first step is to choose reference points. This can be done by dividing the area of interest into cells and choose one reference point for each cell; another very common approach is to choose reference points in the grid with fixed distance between them.

\footnotetext{
* Juraj Machaj, Peter Brida

Department of Telecommunications and Multimedia, Faculty of Electrical Engineering University of Zilina, Slovakia

E-mail: juraj.machaj@fel.uniza.sk
} 


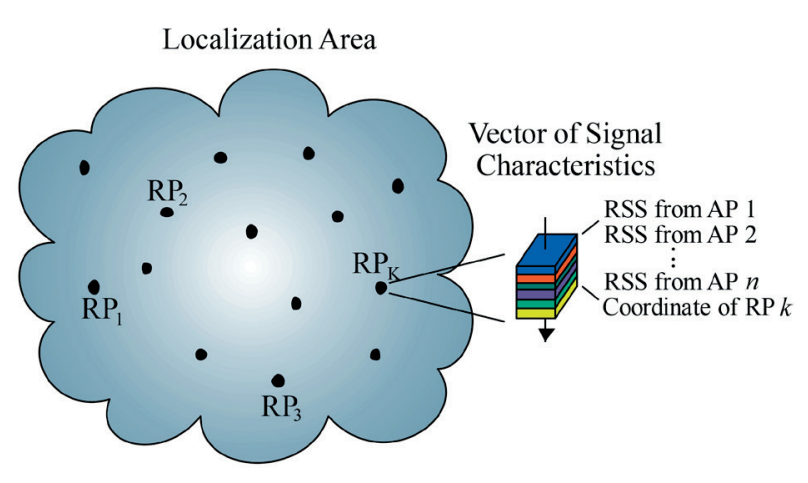

Fig. 1 Construction of radio map

The second step is measurement of RSS values from all Access Points (APs) in the radio range for a certain period of time. The measured RSS vector together with position of the reference point is called fingerprint:

$$
P_{j}=\left(N_{j}, \alpha_{j i}, \theta_{j}\right), \quad j=1,2, \ldots, M,
$$

where $N_{j}$ is the number of $j$-th reference point, $M$ is the number of all reference points, $\alpha_{j i}$ is the vector of RSSI values and parameter $\theta_{j}$ obtains additional information which can be used during the localization phase, e.g. device orientation or information about time [7].

Last step of the offline phase is to save the measured fingerprints from all reference points to the radio map database. The radio map database can be modified or preprocessed before online stage to reduce memory requirements or computational cost of localization algorithm.

\section{B Deterministic Algorithms}

In the deterministic algorithms, the state is assumed to be a non-random vector. The main objective is to compute the position estimate $\hat{x}$, which is a combination of reference points $p_{i}$, that is:

$$
\hat{x}=\sum_{i=1}^{M} \omega_{i} \cdot p_{i} / \sum_{j=1}^{M} \omega_{j},
$$

where all weights $\omega_{i}$ and $\omega_{j}$ are nonnegative [8]. Weights $\omega_{i}$ and $\omega_{j}$ are computed as inverted value of distance between RSS vector from the online phase and vectors stored in the radio map. Euclidean distance is commonly used as a measure between these RSS vectors.

The estimator (2), which keeps the $K$ highest weights and sets the others to zero is called the WKNN (Weighted K-Nearest Neighbor Method) [1]. WKNN with all weights $\omega_{i}=1$ is called the KNN (K-Nearest Neighbor) method. The simplest method, where $K=1$, is called the NN (Nearest Neighbor) method [1]. KNN and the WKNN can perform better than the NN method, particularly with parameter values $K=3$ and $K=4$ [8].

\section{Probabilistic Algorithms}

In the contrast to the deterministic approach in the probabilistic (or statistical) framework the state is assumed to be a random vector [8]. Let us assume that there are $M$ location candidates $\omega_{1}, \omega_{2}, \omega_{3}, \ldots, \omega_{M}$. This means there are $M$ classes from which the most appropriate one is the chosen based on the posteriori probability. These $M$ locations represent reference points where RSS values were measured during the off-line phase. Vector $S$ represents the observed RSS during the on-line stage. The location candidate $\omega$, is chosen in case that its posteriori probability is the highest. The decision rule from Bayes' theorem can be written as:

$$
P\left(\omega_{i} \mid S\right)=\frac{P\left(\vec{S} \mid \omega_{i}\right) P\left(\omega_{i}\right)}{P(\vec{S})},
$$

where posteriori probability $P\left(\omega_{i} \mid S\right)$ is the combination of likelihood $P\left(S \mid \omega_{i}\right)$, prior probability $P\left(\omega_{i}\right)$, and observed evidence $P(S)$. The observed evidence keeps the same during the one positioning process, and the prior probability is assumed to be the same for all over the target environment. Based on this the comparison of the posteriori probability could be considered as the comparison of likelihood.

The likelihood of each location candidate is assumed to have the Gaussian distribution. Therefore, means and standard deviations of each location candidate could be calculated from the sample data. The APs in the environment are assumed to be independent, so the overall likelihood of one location candidate can be calculated by multiplying the likelihoods of all APs:

$P\left(\vec{S} \mid \omega_{i}\right)=P\left(S_{1} \mid \omega_{i}\right) \times P\left(S_{2} \mid \omega_{i}\right) \times P\left(S_{m} \mid \omega_{i}\right)$,

where $m$ is the number of APs and $S_{j}$ represents the RSS from the $j$-th AP [8]. In order to get more accurate results the position can be computed as average of all locations by adopting their posteriori probabilities as weights.

\section{Device calibration methods}

Device calibration methods can be divided into three main groups based on the calibration process. First group called manual calibration methods requires a collection of data at several known locations. Quasi-automatic calibration methods require a collection of data at unknown locations, but complex algorithms are needed to find the mapping of RSS values to reference device. Calibration methods which can automatically obtain fitting parameters based on unsupervised learning are marked as automatic calibration methods.

In the most cases differences in RSS measurement on devices are assumed to be linear and thus can be formulated as:

$$
R S S_{\text {ref }}=a_{x} * R S S_{u}+b_{x},
$$

where $R S S_{u}$ stands for RSS value recorded by an unknown device and $a_{x}, b_{x}$ are parameters of linear transformation between the 
unknown device and reference device. The main goal of the device calibration methods is to find the transformation parameters.

\section{A Manual calibration}

The basic idea for the manual calibration of mobile devices is to measure RSS data at the known calibration points and use these data to find a transformation function between the user device and reference device used for the radio map construction.

Vaupel et al. [9] introduced a manual calibration method which was used during the development of their localization system. Measurements in an anechoic chamber were used to find offset of RSS measured on different devices. RSS measurement offset $b_{x, i}$ for device $x$ and AP $i$ was calculated as:

$$
b_{x, i}=\operatorname{mean}\left(R S S_{r e f, i}\right)-\operatorname{mean}\left(R S S_{x, i}\right) \text {, }
$$

After measurements for all AP in every direction the measurement offsets were combined using all $\mathrm{N}$ offsets:

$$
b_{x}=\frac{1}{N} \sum_{i=1}^{N} b_{x, i}
$$

Measurements from the different directions and different APs were performed due to imperfect omni-directional antenna characteristics of the antennas used in devices and non-linear scaling of the Wi-Fi cards within the measurement range.

In [10] Haeberlen et al. proposed a manual calibration scheme. In this scheme the calibration measurements are performed at several different reference points. The measurements are performed manually and after the device collects a few scans the user must indicate the current reference point on a floor plan of the building. The calibration function is then computed by applying least-squares method.

\section{B Quasi-automatic calibration}

It is clear that even manual calibration methods are effective, but they have the disadvantage of requiring the user to perform measurements at a known position. However, calibration can be performed even without this information, based only on calibration measurements. Calibration methods, which use only calibration measurements, are called quasi-automatic.

In [10] authors propose the use of a calibration method using least-squares algorithm to estimate the transformation function without a known position of the user during calibration measurements. This solution is based on assumption that there is no linear mapping between observations from different positions Based on this assumption it is given that if the chosen reference point is incorrect, the confidence value of Markov localization is low for all reference points. Algorithm must choose reference point in a way that the confidence value is maximized.

Kjaergaad proposed a quasi-automatic calibration method based on least-squares method in [11]. Since in quasi-automatic calibration the measurement positions are unknown the measurements have to be compared to the measurements from all possible reference points. Two methods were proposed to be used in a way to determine position of the user during the calibration measurements. The first method was Bayesian localization with observations from an unknown location and the second method is a comparison method which tries to match the means and standard deviations of the observation with measurements in the radio map.

\section{Automatic calibration}

It is clear that no interaction with the user during the calibration process is a huge advantage of quasi-automatic calibration methods. This is even more obvious in the case that the user is located in the area he is not familiar with. On the other hand, quasi-automatic calibration requires a high number of measurements and use of complex algorithms to calibrate the user device. This was the main reason for development of automatic calibration methods which can calibrate the user device in background during the positioning and navigation process.

The automatic calibration method was proposed in [12]; the authors proposed to compute a log-normalized ratio to reduce the effect of RSS shift between the reference device and the user device. The log-normalized ratio is computed as:

$$
\ln r\left(o_{i}, o_{j}\right)=\log \left(r\left(o_{i}, o_{j}\right)\right)-\log \left(\frac{1}{v_{\max }}\right)
$$

where $r\left(o_{i}, o_{j}\right)$ is the ratio of RSS measured from two access points $\mathrm{AP}_{\mathrm{i}}$ and $\mathrm{AP}_{\mathrm{j}}$ and $v_{\max }$ is the maximum signal strength value. The ratio of RSS is calculated for all pairs of AP with constraint $i>j$ for uniqueness. According to [13] this solution approximates only the ratio, but it cannot handle the offset term in a linear shift function. So it is assumed that the approximation error is increased when the offset term is relatively large.

Unsupervised learning approach, which should improve the approximation error for both ratio and offset terms of the linear shift function, was proposed in [13]. The proposed method can automatically learn a signal-pattern transformation function for any unknown device. The learning procedure can be divided into two general steps. In the first step RSS readings from the tracking device are labeled with rough location estimate using the correlation ratio computed from Pearson product-moment correlation coefficient defined by:

$$
r=\frac{\sum_{i=1}^{k}\left(\left(s_{c}^{i}-\mu\left(s_{c}\right)\right)\left(s_{o}^{i}-\mu\left(s_{o}\right)\right)\right.}{\sqrt{\sum_{i=1}^{k}\left(s_{c}^{i}-\mu\left(s_{c}\right)\right)^{2}} \sqrt{\sum_{i=1}^{k}\left(s_{o}^{i}-\mu\left(s_{o}\right)\right)^{2}}},
$$

where $k$ is the number of APs, $s_{c}$ represents the RSS of the training device from the radio map, $\mu$ is the mean or expected value of a random variable, and $s_{0}$ is the RSS fingerprint from the users device. Pearson correlation is used to measure similarities in the RSS fingerprints. If the value is 0 , the vectors are completely linear independent and if the absolute value of correlation is 1 , there is the best linear dependency between fingerprints. After labeling the RSS data with rough location estimates, four learning algorithms were applied to train the transformation function. 
Koski et al. in [14] proposed a coverage area positioning scheme and calibration method using RSSI histograms. The idea is to create a histogram of the RSS values over a long time interval. The calibration is based on the fact that the shape of histograms for the different mobile devices has similar shapes, thus parameters of distribution such as mean, median or mode are comparable.

In [15] Laoudias et al. proposed an automatic self-calibration method based on the RSS histograms. In this method calibration is performed during the positioning process. In the first step the existing radio map is used to create a histogram of the reference device, from the RSS data stored in the database. Subsequently, when the positioning process starts, the RSS values of currently observed fingerprint are recorded in background in order to create and update the histogram of RSS values for the device. A linear mapping function between histogram from the radio map and positioning process is calculated for the user device. The linear mapping function is then used to transform the measured RSS values to a new fingerprint which is compatible with radio map.

\section{Simulation model and scenarios}

Simulations were performed in a simulation model created in Matlab environment. In the simulation model the RSS is modelled by two independent parts: path-loss and immediate variations of signal strength. Path-loss is based on a multi-floorand-wall propagation model (MFW). The MFW model considers the nonlinear relationship between the cumulative penetration loss and the number of penetrated floors and walls. The total loss $L_{W M F}$ in distance $d$ can be computed from equation:

$L_{M W F}=L_{0}+10 n \log (d)+\sum_{i=1}^{I} \sum_{k=1}^{K_{w i}} L_{w i k}+\sum_{j=1}^{J} \sum_{k=1}^{K_{f j}} L_{f j k}$,

where $L_{0}$ is the path loss in distance of $1 \mathrm{~m}$ in $\mathrm{dB}, n$ is the power decay index, $d$ is the distance between the transceiver and receiver in meters, $I$ is the number of walls types, $K_{w i}$ is the number of traversed walls of category $i, L_{w i k}$ is the attenuation due to wall type $i$ and $k$-th traversed wall in $\mathrm{dB}, J$ stands for the number of floor types, $K_{f j}$ is the number of traversed walls of category $j$ and $L_{f j k}$ represents the attenuation due to wall type $i$ and $k$-th traversed wall in $\mathrm{dB}$.

Immediate variations of the signal strength could be caused by objects motion at the observed area. These variations influence the RSS measurements and add measurement error. The behavior of the variations was derived from experimental measurements. The measurement error is simulated using random variable with lognormal distribution.

The localization was performed at an area of 512 square meters. Reference points were chosen in grid with $2 \mathrm{~m}$ distance between them. The localization area can be seen in Fig. 2. In the figure blue lines represents walls of the building, grey $\mathrm{x}$ represents the position of APs and red dots show the position of the reference points. The position of mobile device was randomly chosen from the area.

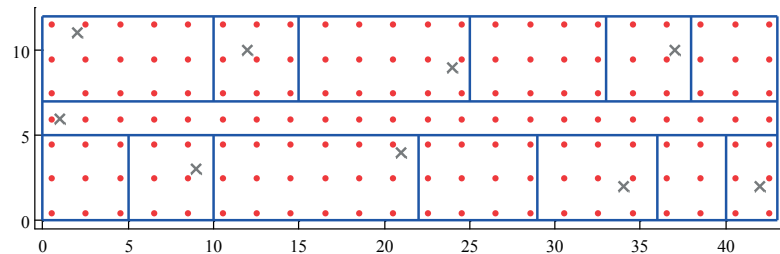

Fig. 2 Localization area

Each fingerprint was created by 20 measurements for a particular AP in very short time on each reference point. The average RSS was calculated from these measurements for every point to eliminate signal fluctuations. Simulations were performed with 1000 independent trials, i.e. for 1000 positions of the mobile device. In the simulations $K=4$ was used for WKNN algorithm.

In the simulations a change of the mobile device was simulated by four different conditions. In the ideal case the RSS values were computed in the same way in both the offline and online phases. Lists of different mobile changes and formulas used to affect the RSS are shown in Table 1.

Device change parameters in simulations

Table 1

\begin{tabular}{|l|l|}
\hline Type of signal change & Relationship to $R S S_{\text {ref }}$ \\
\hline Shift & $R S S_{x}=R S S_{\text {ref }}+G$ \\
\hline Scale & $R S S_{x}=A^{*} R S S_{\text {ref }}$ \\
\hline Shift and scale & $R S S_{x}=A^{*} R S S_{\text {ref }}+G$ \\
\hline Nonlinear & $R S S_{x}=-1\left|\frac{R S S_{\text {ref }}^{2,57}}{R S S_{\text {ref }} * X}\right|$ \\
\hline
\end{tabular}

In the table $R S S_{x}$ represents the RSS received by the uncalibrated device, $R S S_{\text {ref }}$ stands for the RSS received by the reference device, $G$ is the additional gain of antenna in $\mathrm{dB}$ and in simulations was set to $5 \mathrm{~dB}$. Variable $A$ which represents the amplification ratio of the uncalibrated device was set to 0.8 and $X$ is the coefficient which affects nonlinear transformation of the RSS signal and was set to 12 .

In simulation two automatic calibration algorithms were used to remove the impact of device change. The first algorithm was proposed in [12] and in simulation was marked as lognormalization, the second used algorithm was proposed in [15] and we will refer to it as CDF calibration.

\section{Simulation results}

Results of the simulations are shown in this section. In Fig. 3 the results show the impact of different device changes on localization algorithms and calibration techniques. 

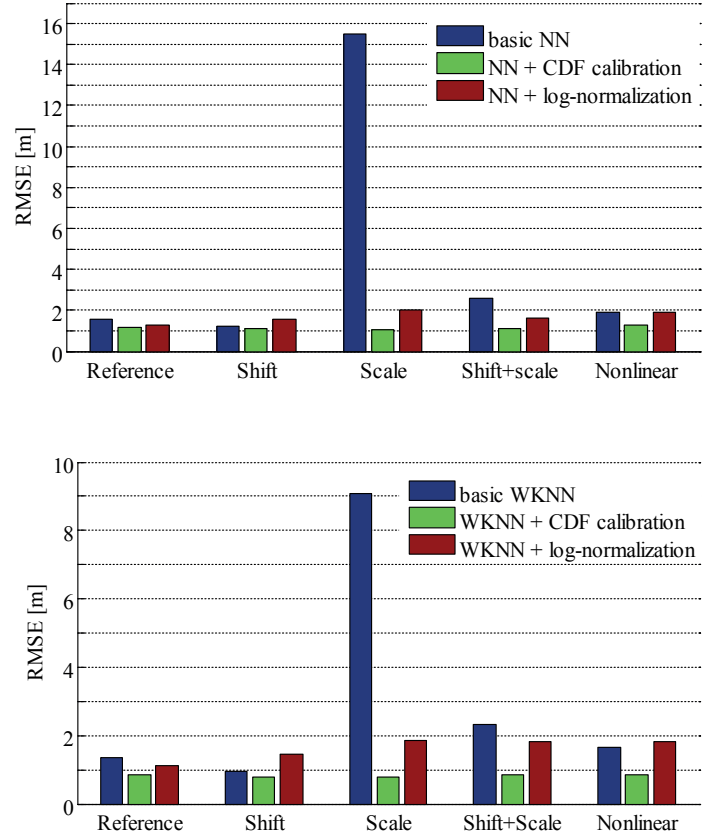

Fig. 3 Impact of device change on calibration and localization accuracy

From the figure it is clear that the worst impact on the positioning accuracy has a scale of device receiver, in other words, differences in antenna gain of different devices. From the results it is also clear that the CDF calibration technique seems to be the most feasible one for a fingerprinting positioning framework. This calibration technique keeps the localization accuracy at the same level in all cases. More detailed results can be seen in Table 2.
From the results it can be seen that the CDF calibration method outperforms calibration via log-normalization in all cases and helps to achieve lower error compared to the fingerprinting positioning without calibration. On the other hand, the lognormalization method achieved the results comparable to the fingerprinting without calibration, but significantly decreased error when the devices have a different scale.

\section{Conclusion}

In the paper the methods for calibration of a mobile device for positioning using a fingerprinting framework were described and compared. The calibration algorithms can be divided into three main groups. The first group consists of manual calibration algorithms, which can achieve the best results, but they have the huge disadvantage in need to perform a large number of measurements at known positions.

In the second group so-called quasi-automatic methods for device calibration can be found. These methods do not need to perform measurements at known positions, but they require a large number of measurements and high complexity algorithms to find a transformation function for the device calibration.

The last group consists of automatic calibration methods. These are running in background of the positioning process. Their main advantage is that there is no need of additional calibration measurements. On the other hand, some of them may need some time to achieve the performance of manual calibration methods. From the simulation it is clear that the automatic calibration technique proposed in [15] significantly reduces the impact of a device change.

\begin{tabular}{|c|c|c|c|c|c|c|c|c|c|}
\hline & & \multicolumn{8}{|c|}{ Localization Error [m] } \\
\hline & & Median & STD & Median & STD & Median & STD & Median & STD \\
\hline \multirow{3}{*}{ Basic } & NN & 1.0685 & 0.7755 & 5.4453 & 14.9938 & 2.2882 & 2.2521 & 1.7675 & 0.9950 \\
\hline & KNN & 0.9433 & 0.5544 & 5.3506 & 9.8282 & 1.9573 & 1.8299 & 1.6546 & 0.8580 \\
\hline & WKNN & 0.8870 & 0.5254 & 5.1950 & 8.7852 & 1.9027 & 1.9213 & 1.5277 & 0.9201 \\
\hline \multirow{3}{*}{ CDF calibration } & NN & 0.9856 & 0.6441 & 0.9637 & 0.6394 & 0.9805 & 0.6641 & 1.1464 & 0.6930 \\
\hline & KNN & 0.8291 & 0.4642 & 0.8490 & 0.4764 & 0.9112 & 0.4981 & 0.8882 & 0.5177 \\
\hline & WKNN & 0.7436 & 0.4296 & 0.7472 & 0.4273 & 0.7924 & 0.4592 & 0.7970 & 0.4594 \\
\hline \multirow{3}{*}{ Log-normalization } & NN & 1.2831 & 1.0080 & 1.7139 & 1.5758 & 2.2116 & 1.6373 & 1.7580 & 1.4022 \\
\hline & KNN & 1.3104 & 0.8800 & 1.6358 & 1.5082 & 2.0608 & 1.7860 & 1.7860 & 1.4086 \\
\hline & WKNN & 1.3190 & 0.8852 & 1.6090 & 1.6399 & 2.0703 & 1.8118 & 1.7765 & 1.3452 \\
\hline \multicolumn{2}{|c|}{ Device change type } & \multicolumn{2}{|c|}{ Shift } & \multicolumn{2}{|c|}{ Scale } & \multicolumn{2}{|c|}{ Shift+scale } & \multicolumn{2}{|c|}{ Nonlinear } \\
\hline
\end{tabular}




\section{Acknowledgements}

This work has been partially supported by the Slovak VEGA grant agency, Project No. 1/0394/13, by

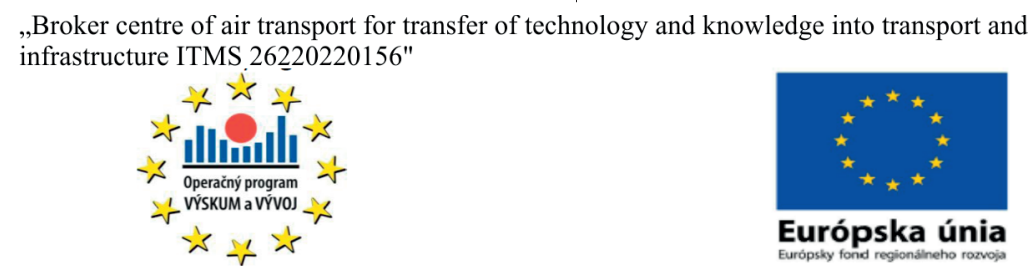

We support research activities in Slovakia/Project is co-financed by EU

and by

Centre of excellence for systems and services of intelligent transport II., ITMS 26220120050 supported by the Research \& Development Operational Programme funded by the ERDF.

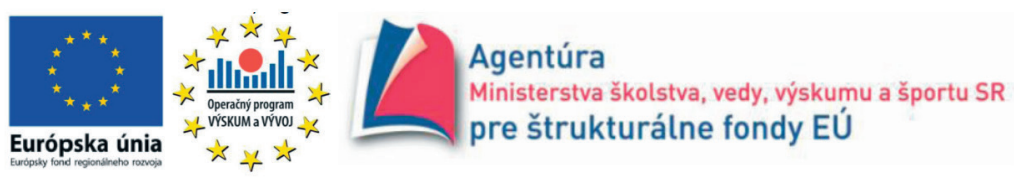

"Podporujeme výskumné aktivity na Slovensku/Projekt je spolufinancovaný zo zdrojov EÚ"

\section{References}

[1] SAHA, S., CHAUHURI, K., SANGHI, D., BHAGWAT, P.: Location Determination of a Mobile Device using IEEE 802.11b Access Point Signals, Wireless Communications and Networking, WCNC 2003. vol. 3. pp. 1987-1992, ISBN: 0-7803-7700-1, 2003

[2] BRIDA, P., MATULA, M., DUHA, J.: Using Proximity Technology for Localization in Wireless Sensor Networks, Communications - Scientific Letters of the University of Zilina, No. 4, vol. 9, 2007, pp. 50-54, ISSN 1335-4205.

[3] KOTZIAN, J., KONECNY, J., KREJCAR, O., LIPPA, T., PROKOP, H., KURUC, M.: The Indoor Orientation of Autonomous Mobile Devices for Increasing Human Perspective, Mobilight Workshops 2010, LNCIST. Springer, Heidelberg, ISBN 978-3-64216643-3, 2010

[4] HAJOVSKY, R., PIES, M.: Complex Measuring System for Longtime Monitoring and Visualization of Temperature and Toxic Gases Concentration, ELEKTRONIKA IR ELEKTROTECHNIKA, No. 6, vol. 122, 2012, pp. 129-132. ISSN 1392-1215.

[5] SROVNAL, V., JR., MACHACEK, Z., SROVNAL, V.: Wireless Communication for Mobile Robotics and Industrial Embedded Devices, $8^{\text {th }}$ Intern. Conference on Networks ICN, Gosier, France. 2009, pp. 1-6, ISBN 978-1-4244-3470-1

[6] CIPOV, V., DOBOS, L., PAPAJ, J.: Cooperative Trilateration-based Positioning Algorithm for WLAN Nodes Using Round Trip Time Estimation, J. of Electrical and Electronics Engineering. No. 1, vol. 4, 2011, pp. 29-34, ISSN 1844-6035, 2011

[7] BRIDA, P., BENIKOVSKY, J.: Radio Map Framework for GSM Positioning, Communications - Scientific Letters of the University of Zilina, No. 4, vol. 11, 2007, pp. 24-27, ISSN 1335-4205, 2007

[8] HONKAVIRTA, V., PERALA, T., ALI-LOYTTY, S., PICHE, R.: A Comparative Survey of WLAN Location Fingerprinting Methods, $6^{\text {th }}$ Workshop on Positioning, Navigation and Communication 2009, WPNC 2009, pp. 243-251, ISBN: 978-1-4244-3292-9, 2009

[9] VAuPEL, T., SEITZ, J., KIEFER, F., HAIMERL, S., THIElECKE, J.: Wi-Fi Positioning: System Considerations and Device Calibration, 2010 intern. conference on Indoor Positioning and Indoor Navigation (IPIN), pp.1-7, 2010

[10] HaEberlen, A., FlanNery, E., LADD, A. M., RUDYS, A., WALlACH, D. S., KAVRAKI, L. E.: Practical Robust Localization over Large-scale 802.11 Wireless Networks, $10^{\text {th }}$ intern. Conference on Mobile computing and networking (MobiCom), pp. 70-84, 2004

[11] KJAERGAARD, M.: Automatic Mitigation of Sensor Variations for Signal Strength Based Location Systems, LoCA'06 Proc. of the $2^{\text {nd }}$ intern. conference on Location- and Context-Awareness pp. 30-47, 2006.

[12] KJAERGAARD, M. B., MUNK, C. V.: Hyperbolic Location Fingerprinting: A Calibration-free Solution for Handling Differences in Signal Strength, VI Annual IEEE Intern. Conference on Pervasive Computing and Communications, pp. 110-116, 2008

[13] TSUI, A. W., CHUANG, Y.-H., CHU, H.-H.: Unsupervised Learning for Solving RSS Hardware Variance Problem in WiFi Localization, Mobile Networks and Applications, No. 5, vol. 14, 2009, pp. 677-691

[14] KOSKI, L., PERALA, T., PICHE, R.: Indoor Positioning using WLAN Coverage Area Estimates, 2010 intern. conference on Indoor Positioning and Indoor Navigation (IPIN), pp.1-7, 2010

[15] LAOUdiAS, C., PICHE, R., PANAYIOTOU, C. G.: Device Signal Strength Self-calibration using Histograms, 2012 Intern. Conference on Indoor Positioning and Indoor Navigation (IPIN), pp.1-8, 2012. 